\title{
Types of Pattern Strabismus and Their Surgical Outcomes after Adjustable Strabismus Surgery
}

\author{
Sana Nadeem ${ }^{1}$ \\ ${ }^{1}$ Department of Ophthalmology, Foundation University Medical College \& Fauji Foundation Hospital, Rawalpindi
}

\begin{abstract}
Purpose: To study the different types of pattern strabismus and their surgical outcomes after adjustable strabismus surgery.
\end{abstract}

Study Design: Prospective, interventional study.

Place and Duration of Study: Eye department, Fauji Foundation Hospital, Rawalpindi (June, 2016 to March, 2019).

Methods: A total of 40 consecutive patients with pattern strabismus were included in the study. After complete orthoptic assessment, the patients underwent either rectus muscle transposition or oblique muscle surgery, by adjustable squint surgery. The pre-operative evaluation of different strabismus patterns, deviations, and postoperative results were measured and analyzed. The amount of postoperative pattern change was measured at 6 weeks postoperatively, to assess the success of the surgeries.

Results: Out of 40 patients, 33 were females and 7 were males. The age ranged from 7 to 69 years (mean 18.05 \pm 9.6 ). All patients were operated by using adjustable suture technique."V" pattern was seen in 26 cases (65\%) and "A" pattern was seen in $12(30 \%)$ cases. The mean preoperative pattern was 13.2 \pm 8.09 PD (prism diopters), and the mean postoperative pattern was $2.05 \pm 2.96 \mathrm{PD}$. Surgical success was defined as the amount of pattern collapse at 6 weeks, the period at which a stable alignment was achieved. The difference between the preoperative and postoperative pattern deviation was statistically significant $(p=0.000)$. Correction of the pattern was seen in $92.5 \%$ of patients at average follow up of $50.87 \pm 14.02$ days.

Conclusions: Pattern Strabismus can be corrected by varying rectus muscle transpositions or oblique muscle surgery during routine adjustable strabismus surgery.

Key Words: Strabismus, $\mathrm{V}$ pattern, A pattern, $\mathrm{Y}$ pattern, $\mathrm{X}$ pattern, lambda pattern, muscle transposition.

How to Cite this Article: Nadeem S. Types of Pattern Strabismus and Their Surgical Outcomes after Adjustable Strabismus Surgery. Pak J Ophthalmol. 2021, 37 (1): 75-82.

Doi: https://doi.org/10.36351/pjo.v37i1.1049

\section{INTRODUCTION}

Strabismus patterns are a common occurrence in routine strabismus patients, although discussed

Correspondence: Sana Nadeem

Department of Ophthalmology, Foundation University Medical College \& Fauji Foundation Hospital, Rawalpindi

Email: sana.nadeem018@gmail.com

Received: April 19, 2020

Accepted: October, 29, 2020 scarcely. These include the frequently occurring "A" and "V" patterns, and also the not so common "Y", " $\lambda$ " (lambda), " $X$ ", and " $\downarrow$ " (arrow) patterns. The " $V$ " pattern was the first of its kind to be described in 1897 , by Duane in a patient with bilateral superior oblique palsy. ${ }^{1.2}$ Years later, in 1948, Urrets-Zavalia described the deviation in upgaze and downgaze, and bought attention to the " $A$ " and "V" patterns, the terms coined by Urist in $1951 .{ }^{1,2}$

Typically, a " $V$ " pattern describes horizontal strabismus, with vertical incomitance in upgaze and 
down gaze of 15 prism diopters (PD) or greater, or more esotropia and less exotropia in down gaze when compared in upgaze. The " $A$ " pattern is defined converse to the "V" pattern with a difference between upgaze and down gaze of 10 PD or more, with more esotropia and less exotropia in upgaze relative to down gaze. Minimal change in down gaze to primary position, but exotropia (divergence) in upgaze, defines a " $Y$ " pattern, seen usually in Duane and Brown syndromes. This is usually caused by bilateral inferior oblique over action in congenital horizontal strabismus. A Lambda (" $\lambda$ ") pattern is converse to the "Y" pattern, in which divergence is maximum between primary position and down gaze. This is observed in bilateral superior oblique over action or inferior rectus under action. An " $X$ " pattern denotes divergence in both upgaze and down gaze, and the exotropia may be controlled in primary position by fusion. This is caused by all four oblique over action. An arrow (" $\downarrow$ ”) pattern, describes maximum convergence in primary and down gaze positions, typically observed in bilateral superior oblique palsy. ${ }^{3}$

Pathophysiological mechanisms for pattern strabismus, though unclear, have been attributed both to mechanical (peripheral) and neural (central) factors; the former occurring as a result of oblique muscle under or overaction, ectopic muscle pulleys or orbital deformities in craniofacial dysostosis, hydrocephalus or plagiocephaly; and the later being caused by loss of fusion with abnormal torsion, abnormal supranuclear circuits or vestibular hypofunction.

Transposition of the horizontal rectii is a reasonable approach for correcting about 15-20 PD of pattern, in the absence of oblique over action; larger amounts require oblique weakening if over action is present; bilateral inferior oblique weakening corrects 15-25 PD, whereas bilateral superior oblique weakening can correct as much as 40 PD. ${ }^{2,3}$

It is important for the strabismus surgeon to recognize both common and rare patterns in strabismus and to correct them accordingly, to provide maximum cosmesis to the patient. The purpose of our study was to identify any patterns occurring in strabismus patients, and to correct them during routine adjustable strabismus surgery.

\section{METHODS}

A total of 40 consecutive patients were included in this study carried out in the Department of Ophthalmology,
Fauji Foundation Hospital, Rawalpindi, a tertiary care teaching hospital affiliated with the Foundation University Medical College. The study was carried out from June, 2016 to March, 2019. Approval from the ethical committee was taken. Horizontal, vertical and complex strabismus cases were included. Patients with previous history of strabismus surgery were also included. All surgeries were performed by the same surgeon. Myasthenia Gravis, pattern less than 5 PD and uncooperative children less than 7 years of age were excluded.

A detailed ocular assessment was done for every case. Best-corrected visual acuity was documented and refractive correction was given to patients prior to the surgery. The preoperative angle of deviation was assessed by the prism cover test with the refractive correction in place, for both near and distance in primary gaze position, as well as in $25^{\circ}$ of upgaze (chin down) and $35^{\circ}$ of down gaze (chin up) ${ }^{1}$, right and left gaze, and head tilt in cases of paralytic strabismus. In certain cases of sensory strabismus with poor fixation, the Krimsky test was used for analysis of the angle or a pen torch was used as a target for near and distance. The distance angle in primary position with refractive correction in place was considered as the angle of deviation in all cases, and the surgical alignment was sought to correct this angle, although at the time of suture adjustment, both near and distance alignment was corrected. Exception to this was accommodative refractive esotropia, for which the near deviation with distance spectacles in place was considered for correction of the alignment.

Measurements were taken by the operating surgeon and repeated one day prior to surgery, to obtain maximum cosmesis. Extraocular motility was checked with muscle over-action graded from +1 to +4 and under-action graded from -1 to -5 . Binocular vision and stereopsis were assessed by the Titmus fly test and Worth-four-dot test, for every case as a routine. A thorough eye examination was performed including fundus examination and intraocular pressures. In case of significant oblique over-action or vertical strabismus, fundus torsion was assessed with the indirect ophthalmoscope.

All surgeries were performed under general anesthesia. A drop of phenylephrine 10\% (Ethifrin ${ }^{\circledR}$ ) was instilled into the conjunctival fornix at the beginning of surgery in each eye. The fornix approach for strabismus surgery was used in every case. Each muscle was hooked, and then secured with a double 

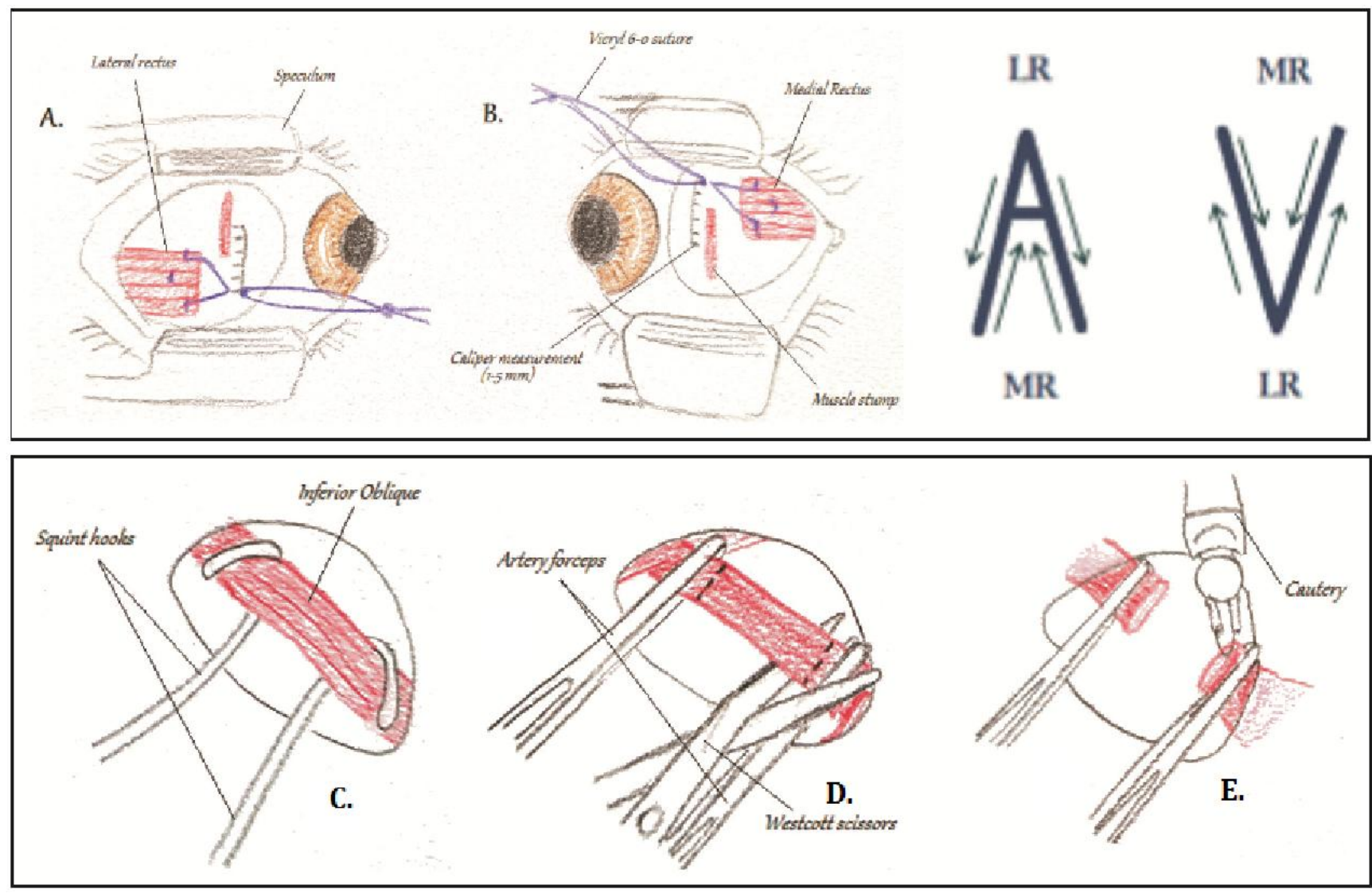

Fig. 1: (A-B) Correction of Pattern For an "A" pattern strabismus (A): Displacement of the lateral rectus away from the apex $5 \mathrm{~mm}$ or half tendon width. (B): Displacement of the medial rectus $5 \mathrm{~mm}$ towards the apex. This is irrespective of whether the rectus muscle is recessed or resected. (C-E): Inferior oblique myectomy. (C): Inferotemporal fornix incision and exposure of inferior oblique with two squint hooks.

(D): Clamping of each end with artery forceps and cutting it between them. (E): Cautery to cut ends of muscle.

armed 6-0 vicryl, which was passed through the sclera at its insertion, or transposed above or below the insertion in case of "A" or "V" patterns, in a "hangback' fashion. The medial rectii were transposed towards the apex of the pattern, and the lateral rectii were transposed away from the apex of the pattern (Figure 1 A-B) (Figure 2: A-D).

The amount of transposition varied from $1 \mathrm{~mm}$ to $5 \mathrm{~mm}$, the latter being the standard half tendon width. This depended upon the amount of pattern noted and increased with increasing pattern. In case of significant inferior oblique over-action or DVD (Dissociated vertical deviation), inferior oblique myectomy (Figure $1 \mathrm{C}-\mathrm{E})$ or anteriorization respectively, were done to correct the pattern.

The recessed muscles were mostly placed for adjustable purpose, with the required recession held in place by Guyton's modification of the sliding noose knot, which was fashioned with a 6-0 vicryl suture. The amount of 'hang-back' recession was calculated for each patient using standard tables. The traction suture for holding the sclera for postoperative adjustment was created with ethibond 5-0 in every case. For the non-adjustable recessions, the muscle was tied and allowed to 'hang-back' from its insertion, with the required amount of recession calculated as required. For resections, the amount of resection was overcorrected by $2 \mathrm{~mm}$, and allowed to 'hang-back' for this distance, to be adjusted if required postoperatively. At least one muscle was kept on an adjustable sliding noose knot per case; with complex strabismus, all muscles were kept on adjustable sutures.

All patients were assessed for alignment and final adjustment at least 1 hour or more after surgery, in the recovery room, to allow for the effects of general anesthesia to wear off. The eyes were anesthetized topically with Alcaine ${ }^{\circledR}$ (proparacaine hydrochloride $0.5 \%$ ) eye drops. The patients were assessed with the cover-uncover test at distance and near, with a torch 
light for distance if the vision was blurred, or a distance readable target, and for near an accommodative target was used. If the alignment was satisfactory, with no movement on cover testing, the sutures were tied off in their existing position, held in place by the sliding noose, which was removable after tying the ends of the muscle sutures. Thereafter, the traction knot was cut, and the conjunctiva was sutured with at least one 6-0 vicryl suture. The final tying off point was orthotropia or maximum possible undercorrection as required. In cases of exotropia, the goal was orthotropia or mild esotropia. In cases of esotropia, the goal was either orthotropia, if achieved, or slight under-correction. The transpositions were not adjustable, but were predetermined, and so were the inferior oblique weakening procedures. The superior oblique muscle was not operated upon in any case. In five cases of small degrees of patterns, only routine adjustable surgery was performed.

The alignment was noted postoperatively, the next day, at 2 weeks follow up, and then at 6 weeks after surgery. The patients were given postoperative topical steroids and antibiotic drops twice a day and ointment at night for a minimum of 2 weeks.

Surgical success or primary outcome measure was defined in terms of amount of collapse of the pattern as compared to pre-operative pattern, at the end of at least 6 weeks after surgery. This is the time at which a stable alignment is achieved and this usually correlates with long-term success. Although both horizontal, vertical and complex strabismus were included in our study, the horizontal alignments preoperatively and postoperatively, were mainly assessed except for 2 cases of monocular elevation deficiency, in which the hypotropia was assessed for success of adjustable strabismus surgery. Horizontal deviations were assessed and analyzed for pattern strabismus collapse after surgery.

The results were noted, tabulated and analyzed using the SPSS version 20. Frequencies were calculated for age, gender, type and pattern of strabismus, surgical procedure performed, as well as the follow up period. The difference between the preoperative and postoperative deviation and patterns was analyzed by the Wilcoxon Signed Ranks test, with a significance of less than 0.05 .

\section{RESULTS}

A total of 40 consecutive patients with pattern strabismus presenting to us were included in this study. The mean age was $18.05 \pm 9.6$ years (range 7 $69)$. There were $33(82.5 \%)$ females and the 7 males, $(\mathrm{n}=7,17.5 \%)$. The type of deviation in the majority of patients was exotropia in $17(42.5 \%)$ cases (Table 1). The pre-operative and post-operative patterns are detailed in Table 2, with the majority being "V" patterns in $26(65 \%)$ cases and "A" pattern in 12 (30\%) cases (Figure 2).

Table 1: Type of Deviation.

\begin{tabular}{|c|c|c|c|c|c|c|c|c|c|}
\hline \multirow{2}{*}{ Type of Deviation } & \multirow{2}{*}{ Frequency(Percent) } & \multicolumn{4}{|c|}{ Pattern Preoperative } & \multicolumn{4}{|c|}{ Pattern Postoperative } \\
\hline & & $\mathbf{V}$ & $\mathbf{A}$ & $\mathbf{X}$ & $\mathbf{Y}$ & $\mathbf{V}$ & $\mathbf{A}$ & $\mathbf{Y}$ & None \\
\hline Exotropia & $17(42.5)$ & 15 & 2 & 0 & 0 & 6 & 3 & 2 & 6 \\
\hline Esotropia & $9(22.5)$ & 3 & 6 & 0 & 0 & 0 & 4 & 0 & 5 \\
\hline Exotropia \& DVD & $2(5.0)$ & 1 & 0 & 1 & 0 & 1 & 0 & 1 & 0 \\
\hline Esotropia \& DVD ${ }^{\Phi}$ & $2(5.0)$ & 1 & 1 & 0 & 0 & 1 & 0 & 0 & 1 \\
\hline Exotropia \& Hypertropia & $4(10.0)$ & 4 & 0 & 0 & 0 & 1 & 0 & 0 & 3 \\
\hline Esotropia \& Hypertropia & $1(2.5)$ & 0 & 1 & 0 & 0 & 0 & 1 & 0 & 0 \\
\hline Esotropia \& Hypotropia & $4(10.0)$ & 2 & 1 & 0 & 1 & 1 & 1 & 0 & 2 \\
\hline Exotropia \& Hypotropia & $1(2.5)$ & 0 & 1 & 0 & 0 & 0 & 1 & 0 & 0 \\
\hline Horizontal & $18(45.0)$ & 12 & 6 & 0 & 0 & 4 & 5 & 2 & 7 \\
\hline Horizontal and Vertical & $6(15.0)$ & 5 & 0 & 1 & 0 & 2 & 1 & 0 & 3 \\
\hline Complex ${ }^{\epsilon}$ & $10(25.0)$ & 4 & 5 & 0 & 1 & 3 & 4 & 0 & 3 \\
\hline Horizontal \& Complex ${ }^{\epsilon}$ & $4(10.0)$ & 3 & 1 & 0 & 0 & 1 & 0 & 0 & 3 \\
\hline Horizontal, Vertical \& Complex ${ }^{\epsilon}$ & $2(5.0)$ & 2 & 0 & 0 & 0 & 0 & 0 & 1 & 1 \\
\hline
\end{tabular}

$\Phi$ Dissociated vertical deviation

$€$ Sensory, Monocular elevation deficit, paralytic strabismus, nystagmus or DVD 
Table 2: Pattern of Strabismus.

\begin{tabular}{lcclcc}
\hline Pattern & $\begin{array}{c}\text { Pre-Operative Patterns } \\
\text { Frequency }\end{array}$ & Percent & Pattern & Post-Operative Residual Patterns \\
F pattern & 26 & 65.0 & None & 17 & Percent \\
A pattern & 12 & 30.0 & V pattern & 10 & 42.5 \\
X pattern & 1 & 2.5 & A pattern & 10 & 25.0 \\
Y Pattern & 1 & 2.5 & Y Pattern & 3 & 25.0 \\
\hline
\end{tabular}
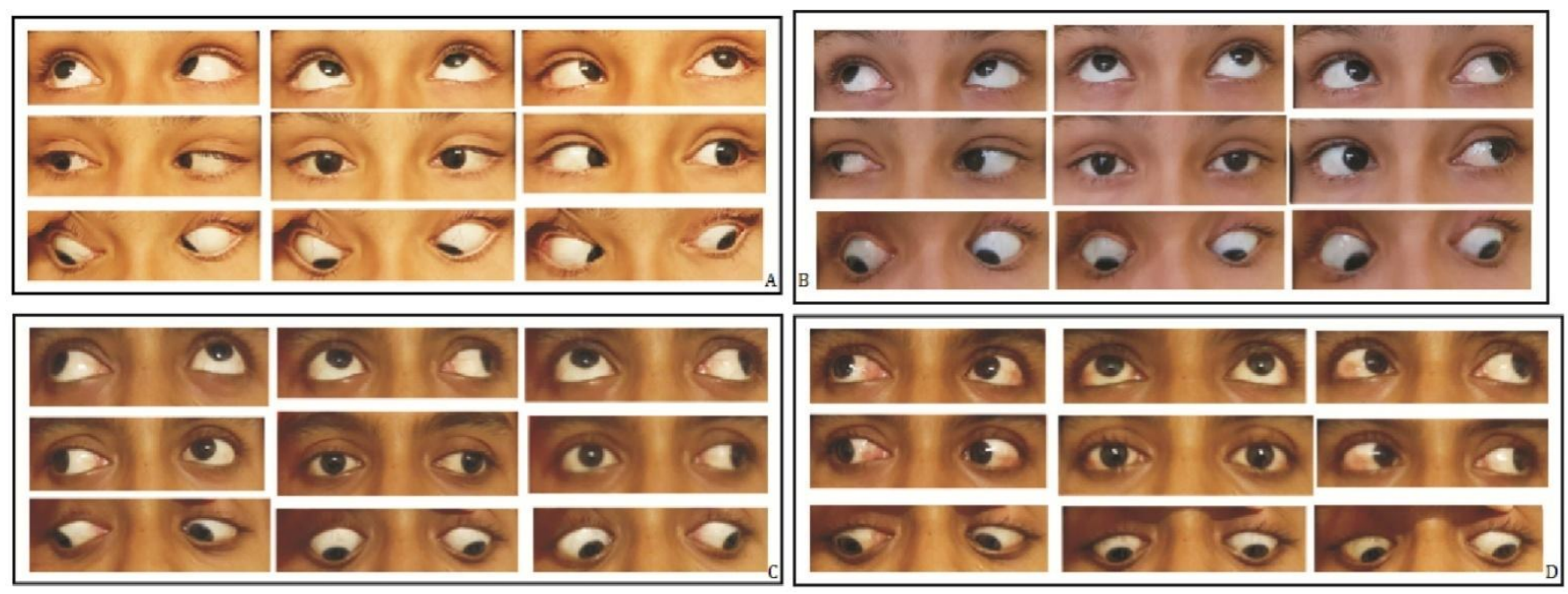

Fig. 2: A. Left Esotropia of 80 PD with an "A" pattern of 25 PD, with bilateral Duane syndrome Type I. B. Post-operative appearance at 6 weeks, after bi-medial recessions $7 \mathrm{~mm}$ OD and $10 \mathrm{~mm}$ OS and $5 \mathrm{~mm}$ upward transposition of each. C. Alternate exotropia of 70 PD and a "V" pattern of 32 PD; managed by bilateral lateral rectus recessions of $7 \mathrm{~mm}$ and a right medial rectus resection of $6 \mathrm{~mm}$, and bilateral inferior oblique myectomies. D. Postoperative appearance.

Table 3: Details of Surgical Procedures.

\begin{tabular}{|c|c|c|c|c|c|}
\hline \multicolumn{2}{|c|}{ Surgical Procedures for Pattern deviations } & \multicolumn{4}{|c|}{ Surgical Procedures for Horizontal and Vertical Deviation } \\
\hline Surgery for Pattern Correction & $\begin{array}{c}\text { Frequency } \\
\text { (Percentage) }\end{array}$ & $\begin{array}{c}\text { Horizontal Muscle } \\
\text { Surgery }\end{array}$ & $\begin{array}{c}\text { Frequency } \\
\text { (Percent) }\end{array}$ & Vertical Muscle Surgery & $\begin{array}{c}\text { Frequency } \\
\text { (Percent) }\end{array}$ \\
\hline No surgery performed & $5(12.5)$ & BLRc $^{\| l}$ & $10(25)$ & None & $22(55)$ \\
\hline $1 \mathrm{~mm}$ transposition $^{\beta}$ & $1(2.5)$ & $\mathrm{BMRc}^{\S}$ & $10(25)$ & Unilateral IO ${ }^{\alpha}$ myectomy & $4(10)$ \\
\hline $2 \mathrm{~mm}$ transposition ${ }^{\beta}$ & $5(12.5)$ & $\mathrm{BMRs}^{¥}$ & $1(2.5)$ & Bilateral $\mathrm{IO}^{\alpha}$ myectomies & $7(17.5)$ \\
\hline $3 \mathrm{~mm}$ transposition ${ }^{\beta}$ & $3(7.5)$ & $\mathrm{MRc}^{\mathrm{a}}+\mathrm{LRs}^{x}$ & $1(2.5)$ & $\mathrm{IO}^{\alpha}$ anteriorization & $1(2.5)$ \\
\hline $4 \mathrm{~mm}$ transposition ${ }^{\beta}$ & $1(2.5)$ & $\mathrm{MRs}^{\emptyset}+\mathrm{LRc}^{\mathrm{H}}$ & $10(25)$ & $\mathrm{SR}^{\Omega}$ transposition & $1(2.5)$ \\
\hline $4.5 \mathrm{~mm}$ transposition ${ }^{\beta}$ & $1(2.5)$ & $\mathrm{BLRc}^{\mathbb{I}}+\mathrm{MRs}^{\varnothing}$ & $2(5)$ & Bilateral IO myectomies $+\mathrm{SRc}^{2}$ & $1(2.5)$ \\
\hline $5 \mathrm{~mm}(1 / 2$ tendon $)$ transposition ${ }^{\beta}$ & $10(25)$ & $\mathrm{BMRc}^{\S}+\mathrm{LRs}^{\times}$ & $2(5)$ & $\mathrm{IRc}^{7}+\mathrm{SRc}^{2}$ & $3(7.5)$ \\
\hline Unilateral IO myectomy & $4(10)$ & $\mathrm{MRc}^{\alpha}$ & $2(5)$ & $\operatorname{IRc}^{*}$ & $1(2.5)$ \\
\hline Bilateral IO myectomy & $9(22.5)$ & $\mathrm{LRc}^{\mathrm{H}}$ & $1(2.5)$ & & \\
\hline IO anteriorization & $1(2.5)$ & $\mathrm{LRc}^{\mathrm{H}}+\mathrm{MRs}^{\emptyset}+\mathrm{MRc}^{\emptyset}$ & $1(2.5)$ & & \\
\hline
\end{tabular}

$\alpha$ Inferior oblique

$\beta$ Transposition of medial rectus towards apex of pattern and lateral rectus towards empty space of pattern

II Bilateral recessions

a Unilateral medial rectus recession

H Unilateral lateral rectus recession

$\Sigma$ Superior rectus recession

\author{
$\S$ Bimedial recessions \\ $\times$ Unilateral lateral rectus resection \\ $\alpha$ Inferior oblique \\ $\lambda$ Inferior rectus recession
}

$¥$ Bimedial resections

$\emptyset$ Unilateral medial rectus resection $\Omega$ Superior rectus
Table 3 outlines a list of surgeries performed for correction of the patterns and other primary deviations. The average number of muscles operated upon for each patient were $2.92 \pm 1.09$ (Range 2-6).
The mean pre-operative pattern deviation was 13.2 \pm 8.09 PD (prism diopters) with a range from 5 to 35 PD. The mean post-operative pattern deviation was $2.05 \pm 2.96$ PD [Range 0-12 PD] at 6 weeks. Surgical 
success or collapse of the pattern was seen in $92.5 \%$ of patients. Five cases of small patterns in which no surgery for the pattern was undertaken were also observed for post-operative pattern collapse; in one case, the pattern persisted, in three cases, it disappeared completely, and in another one case, it increased. Inferior oblique anteriorization to treat DVD, accentuated the pattern slightly, but treated the DVD. Inferior oblique myectomy was effective in all cases.

The Wilcoxon signed ranks test was used to analyze the pre-operative pattern and post-operative pattern collapse, and the difference between the two was found to be statistically significant $[\mathrm{p}=0.000]$.

The mean preoperative deviation was 49.02 \pm 17.63 PD (Range 20-83 PD), and the mean postoperative deviation was $2.53 \pm 4.42$ PD [Range 020 PD]. The Wilcoxon test was also used to compare the preoperative and postoperative deviation, to assess for success of our adjustable strabismus technique, which was highly significant $(\mathrm{p}=0.000)$.

The success rate of adjustable strabismus surgery in the short term (6 weeks postoperatively) for these patients was also $92.5 \%$, in whom a postoperative deviation within $\leq 10$ PD of orthotropia was achieved. The average follow-up was $50.87 \pm 14.02$ days (Range 38-94 days).

\section{DISCUSSION}

Alphabet patterns in strabismus are important aspects of strabismus management that need to be assessed carefully during workup of routine strabismus patients. Ignoring these patterns may lead to under or over corrections, with subsequent disappointment for both patient and surgeon. ${ }^{4}$

Around $12-87 \%$ of strabismus patients have an "A" or "V" pattern. ${ }^{1,2,4}$ The mechanism of pattern strabismus is still unclear. Many theories have been put forward regarding its etiology. The most popular theory to date was proposed by Knapp, who listed oblique muscle dysfunction as the prime cause, with overacting inferior obliques in " $\mathrm{V}$ " patterns, and overacting superior obliques in "A" patterns. ${ }^{2}$ Deng et $\mathrm{al}^{5}$ supported this theory by strongly correlating fundus intorsion, and superior oblique over action with "A" patterns, and fundus extorsion and inferior oblique over action with "V" patterns. Kushner" suggested the role of ocular torsion as a cause of these patterns, as it can cause vertical displacement of rectus insertions. Craniofacial dysmorphisms, orbital pulley heterotopias or laxity, as well palpebral fissure slanting can contribute to such patterns. ${ }^{2}$ They can also arise iatrogenically. ${ }^{7}$

There are numerous studies where patterns of strabismus have been studied and a variety of surgical procedures have been employed over the years for their correction. Conventionally, in the absence of oblique over action, horizontal rectii are displaced, with medial rectus towards the apex of the pattern and lateral rectus away ${ }^{8-11}$ Monocular surgery with vertical displacement of the horizontal rectii was studied by Metz and Schwartz ${ }^{8}$ with satisfactory results, and no subsequent torsional symptoms. These are good options for small patterns. ${ }^{9} \mathrm{Oya}^{10}$ et al showed that the amount of correction corresponds to the size of the pattern. We found that varying horizontal rectus transpositions during routine adjustable strabismus surgery is very effective in reducing pattern strabismus postoperatively. Depending upon the size of the pattern, we varied our transpositions from $1 \mathrm{~mm}$ to $5 \mathrm{~mm}$, and all the horizontal rectus muscles involved during surgery were transposed (Range 2-3). We believe that patterns of less than 5 PD may resolve on their own, and may not need transposition, and did not include them in our study. Some surgeons have opted out horizontal muscle transposition in "sub- $A$ " or "sub-V" patterns in exotropia, and claimed that routine muscle surgery corrected these patterns post operatively. Sub-patterns were less than the classical definition of "A" or "V" patterns i.e. < 10 PD or $<15$ PD respectively. We have observed that leaving sub-patterns as such with no surgery performed for their correction is risky, and patterns more than 5 PD may not correct without transposition, and may even increase after standard strabismus surgery.

Insertion slanting recessions or resections have historically been described for the treatment of these patterns, but were not considered to be of much effect. ${ }^{2,3,12}$ Recently, these procedures are again being advocated for treatment of "A" or "V" patterns with comparable success with transposition. ${ }^{12-13}$ We did not slant any muscle insertion during our study. Van der Meulen-Schot et $\mathrm{al}^{14}$ demonstrated partial tenotomy of the horizontal rectiias the effective treatment option for pattern strabismus.

In case of significant inferior oblique muscle over action as a cause of pattern strabismus or if the pattern is very large, weakening procedures like graded recession, myectomy or anterior transposition are good 
options. $^{15-17}$ Minguini $^{18}$ et al compared anterior transposition to graded recession and found equal effectiveness. Kumar ${ }^{19}$ et al advocated hang-back inferior oblique recession for V-pattern therapy. A bilateral oblique weakening procedure was expected to give greater success than unilateral surgery. ${ }^{20} \mathrm{We}$ preferred myectomy of the inferior oblique, in case of "V" patterns with significant inferior oblique over action, and we found it immensely effective in all 13 $(32.5 \%)$ cases in collapsing the pattern. Inferior oblique anteriorization, however may not be as effective, as in our only case of DVD, which increased the "V" pattern slightly. Akar" et al demonstrated effectiveness of graded anterior transposition of the inferior obliques on "V" pattern. Caldeira et $\mathrm{al}^{22}$ noted the development of a vertical imbalance in $1 / 5$ of the cases, after bilateral graded inferior oblique recession for "V" esotropia. Superior oblique tuck ${ }^{2}$ may also be used to treat "V" pattern.

In case of "A" patterns with large bilateral superior oblique over action, posterior $7 / 8$ tenectomy is successful in pattern collapse of all magnitudes as reported by Ranka and coworkers. ${ }^{23}$ Nasal tenectomy, disinsertion, tendon spacer or graded recession have all been tested and tried. ${ }^{24}$ However, caution is advised in superior oblique surgery, as it may induce bothersome torsional diplopia. Ron ${ }^{25}$ et al prefer Ztenotomy combined with horizontal muscle surgery.

In case of "X", "Y", “ $\lambda$ ”, or " $\downarrow$ " patterns, similar principles of surgery are indicated depending on rectus or oblique over action, and these can be corrected accordingly.

Strength of our study was that this study had a very good success rate both for pattern correction and for the primary deviation. Classical patterns as well as sub-patterns have been included and managed. The patients were all happy and no further repeat surgery was needed in any case, either to correct residual strabismus or pattern.

Limitations of our study are that it reflects the preference of a single center only, and that other centers may differ in their techniques for pattern correction. Torsional outcomes of pattern correction were not noted by us, nor reported by any patient. Superior oblique surgery was not done in any case. Future work required is a multi-centered, large scale study for a more comprehensive analysis of pattern correction.

\section{CONCLUSION}

Rectus muscle transpositions can be varied to treat alphabet pattern strabismus, along with oblique muscle surgery, during adjustable strabismus surgery. With good techniques, patterns can be successfully eliminated to enhance the postoperative cosmetic outlook.

\section{Ethical Approval}

The study was approved by the Institutional review board/Ethical review board. (217-1/FF/FUMC/ERC)

\section{Conflict of Interest}

Authors declared no conflict of interest.

\section{REFERENCES}

1. Kekunnaya R, Mendonca T, Sachdeva V. Pattern strabismus and torsion needs special surgical attention. Eye (London). 2015; 29(2): 184-190.

2. Hoyt CS, Taylor D. Pediatric Ophthalmology and Strabismus. Fourth Edition. Elsevier; China, 2013: p 812-821.

3. Wright KW, Spiegel PH, Thompson L. Alphabet patterns and oblique muscle dysfunction. In: Handbook of Pediatric Strabismus and Amblyopia. Springer; USA, 2006: p 285-322.

4. Sekeroglu HT, Turan KE, Uzun S, Sener EC, Sanac AS. Horizontal muscle transposition or oblique muscle weakening for the correction of V pattern? Eye, 2014; 28: 553-556.

5. Deng H, Irsch K, Gutmark R, Phamonvaechavan P, Foo FY, Anwar DS, et al. Fusion can mask the relationships between fundus torsion, oblique muscle over action/under action, and A- and V-pattern strabismus. J AAPOS. 2013; 17 (2): 177-183.

6. Kushner BJ. Effect of ocular torsion on A and V patterns and apparent oblique muscle over action. Arch Ophthalmol. 2010; 128 (6): 712-718.

7. Khawam E, Jaroudi M, Abdulaal M, Massoud V, Alameddine R, Maalouf F. Major Review: Management of strabismus vertical deviations, A- and V-patterns and cyclotropia occurring after horizontal rectus muscle surgery with or without oblique muscle surgery. Binocul Vis Strabolog Q Simms Romano. 2013; 28 (3): 181-192.

8. Metz HS, Schwartz L. The treatment of A and V patterns by monocular surgery. Arch Ophthalmol. 1977; 95 (2): 251-253.

9. Garrido JT, Goldchmit M, Souza-Dias CR. Vertical transposition of medical rectus muscles for correction of A-pattern anisotropia. Binocul Vis Strabismus Q. 2004; 19 (4): 207-215. 
10. Oya Y, Yagasaki T, Maeda M, Tsukui M, Ichikawa K. Effects of vertical offsets of the horizontal rectus muscles in V-pattern exotropia without oblique dysfunction. J AAPOS. 2009 Dec; 13 (6): 575-577.

11. Awadein A, Fouad HM. Management of large Vpattern exotropia with minimal or no inferior oblique overaction. J AAPOS. 2013; 17 (6): 588-593.

12. Kushner BJ. Insertion slanting strabismus surgical procedures. Arch Ophthalmol. 2011; 129 (12): 16201625.

13. Mostafa AM, Kassem RR. A comparative study of medial rectus slanting recession versus recession with downward transposition for correction of V-pattern esotropia. J AAPOS. 2010; 14 (2): 127-131.

14. Van-der-Meulen-Schot HM, van-der-Meulen SB, Simonsz HJ. Caudal or cranial partial tenotomy of the horizontal rectus muscles in $\mathrm{A}$ and $\mathrm{V}$ pattern strabismus. Br J Ophthalmol. 2008; 92 (2): 245-251.

15. Monteiro-de-Carvalho KM, Minguini N, Dantas FJ, Lamas P, Jose NK. Quantification (grading) of inferior oblique muscle recession for $\mathrm{V}$-pattern strabismus. Binocul Vis Strabismus Q. 1998; 13 (3): 181-184.

16. Polati M, Gomi C. Recession and measured, graded anterior transposition of the inferior oblique muscles for V-pattern strabismus: outcome of 44 procedures in 22 typical patients. Binocul Vis Strabismus Q. 2002; 17 (2): 89-94.

17. Caldeira JA. Some clinical characteristics of V-pattern exotropia and surgical outcome after bilateral recession of the inferior oblique muscle: a retrospective study of 22 consecutive patients and a comparison with Vpattern esotropia. Binocul Vis Strabismus Q. 2004: 19 (3): $139-150$.

18. Minguini N, de-Carvalho KM, de Araújo L, Crosta C. Anterior transposition compared to graded recession of the inferior oblique muscle for V-pattern strabismus. Strabismus. 2004; 12 (4): 221-225.
19. Kumar K, Prasad HN, Monga S, Bhola R. Hangback recession of inferior oblique muscle in $\mathrm{V}$-pattern strabismus with inferior oblique over action. J AAPOS. 2008; 12 (4): 401-404.

20. Nowakowska O, Broniarczyk-Loba A, Loba PJ. The reduction of $\mathrm{A}-\mathrm{V}$ patterns with oblique muscles overaction in unilateral and bilateral surgery. Kiln Oczna. 2008; 110 (10-12): 361-363.

21. Akar S, Gökyiğit B, Yilmaz OF. Graded anterior transposition of the inferior oblique muscle for $\mathrm{V}$ pattern strabismus. J AAPOS. 2012; 16 (3): 286-290.

22. Caldeira JA. V-pattern esotropia: a review; and a study of the outcome after bilateral recession of the inferior oblique muscle: a retrospective study of 78 consecutive patients. Binocul Vis Strabismus Q. 2003; 18 (1): 3548.

23. Ranka MP, Liu GT, Nam JN, Lustig MJ, Schwartz SR, Hall LS. Bilateral posterior tenectomy of the superior oblique muscle for the treatment of A-pattern strabismus. J AAPOS. 2014; 18 (5): 437-440.

24. Ung T, Raoof N, Burke J. Effect of superior oblique weakening on vertical alignment and horizontal and vertical incomitance in patients with A-pattern strabismus. J AAPOS. 2011; 15 (1): 9-13.

25. Ron Y, Snir M, Axer-Seigel R, Friling R. Z-tenotomy of the superior oblique tendon and the horizontal rectus muscle surgery for A-pattern horizontal strabismus. J AAPOS. 2009; 13 (1): 27-30.

\section{Author's Designation and Contribution}

Sana Nadeem; Assistant Professor: Concepts, Design, Literature search, Data acquisition, Data analysis, Statistical analysis, Manuscript preparation, Manuscript editing, Manuscript review. 